\title{
EPIDEMIOLOGIA
}

\section{Infecciones en cirugía de artroplastía de cadera: Resultados del sistema de vigilancia epidemiológica de las infecciones intrahospitalarias. 1996-1999}

FERNANDO OTAIZA O'R. ${ }^{1}$ y E.U. POLA BRENNER F. ${ }^{1}$

\author{
INFECTIONS IN HIP ARTHROPLASTY: RESULTS OF THE \\ NOSOCOMIAL INFECTIONS SURVEILLANCE SYSTEM OF CHILE. \\ 1996-1999
}

Epidemiological surveillance of nosocomial infections in hip arthroplasty was established in chilean hospitals in 1996. Between 1996 and 1999 the surveillance system reported, in 28 hospitals, 134 infections in 3.889 operations, with a cummulative rate of $3.5 \%$. Epidemiological surveillance in Chile does not differentiate infections of the surgical wound from infections of the hip replacement itself, and the period of follow up is during hospital stay after surgery. The most frequent etiological agents were non fermentative Gram negative bacilli (33.3\%), mainly Acinetobacter baumannii. Gram positive cocci represented $23.8 \%$ of the etiologies. Hospitals with higher mean number of operations had lower rates (5.0\% in hospitals with low surgical volume and $2.9 \%$ in hospitals with higher volume). The findings of the surveillance system suggest some risk factors that require further research, such as the duration of preoperative hospital stay and experience of the surgical center.

Key words: Nosocomial infection, Arthroplasty, Epidemiological surveillance.

\section{INTRODUCCION}

El reemplazo de cadera es una intervención frecuente que permite la recuperación rápida de pacientes con fracturas o graves afecciones crónicas de la articulación coxofemoral. La intervención consiste en la inserción de un componente que reemplaza la cabeza del fémur (artroplastía parcial) y en ocasiones, también de un componente acetabular (artroplastía to- tal). La infección post operatoria constituye una complicación grave en estas intervenciones. En la experiencia nacional, la morbilidad en términos de prolongación de la estadía hospitalaria, comparando pacientes infectados con no infectados, pareados por edad y sexo, fue en promedio de 27,6 días (Nercelles P, comunicación personal). La presencia del implante funciona como un cuerpo extraño que genera mayor susceptibilidad a las infecciones; así, se

\footnotetext{
${ }^{1}$ Unidad de Infecciones Intrahospitalarias, División de Salud de las Personas, Ministerio de Salud de Chile.
} 
requiere menor inóculo microbiano, especies menos virulentas pueden ocasionar infecciones y se dificulta el tratamiento. Es difícil erradicar las bacterias adheridas a la prótesis y con frecuencia se debe remover la prótesis con graves consecuencias funcionales, temporales o definitivas. En las series clínicas descritas en países industrializados los agentes etiológicos más frecuentes son Staphylococcus aureus y Staphylococcus coagulasa negativa sumando 46 a $64 \%$ del total. ${ }^{1}$ Los microrganismos se pueden introducir durante el acto operatorio o por vía hematógena. En el primer caso generalmente las infecciones se manifiestan antes de 12 semanas desde la operación representando aproximadamente $40 \%$ del total. Las infecciones subagudas se pueden presentar hasta dos años después de la intervención quirúrgica y representan $45 \%$ del total; con frecuencia son el resultado de una siembra vía hematógena. Las infecciones que se manifiestan después de dos años constituyen menos del $2 \% .{ }^{1}$ Las tasas globales de infección intrahospitalaria (IIH) de prótesis de cadera, desde su instalación hasta la muerte del paciente o retiro de la prótesis, ${ }^{1,2}$ varían entre 0,5 y $2 \%$. Antes de la década de los ' 90 s se alcanzaron cifras superiores a $4 \%$ en pacientes operados, con muchos factores de riesgo o durante brotes epidémicos. ${ }^{2-4}$ En Chile hay escasa información sobre este tema, sólo un estudio con una tasa de $1,1 \%$ de infecciones profundas, sin descripción de los microorganismos causales. ${ }^{5}$

Los factores de riesgo de infección conocidos son: artritis reumatoidea, diabetes mellitus, edad avanzada, presencia de focos infecciosos distales, cirugía previa de cadera, ${ }^{6,7}$ antibioprofilaxis inadecuada, calidad de la técnica y abordaje quirúrgicos, reintervenciones, estadía preoperatoria prolongada, ${ }^{3}$ gravedad del paciente y duración del tiempo operatorio. ${ }^{2}$ No han sido establecidos como factores de riesgo la obesidad, la terapia inmunosupresora, la desnutrición $^{6-9}$ ni el uso de cemento para fijar el implante. ${ }^{10}$ Existe una relación inversa entre el volumen de artroplastías realizadas y la frecuencia de complicaciones, entre ellas las IIH, centros o profesionales que realizan más intervenciones tienen menores tasas de infección. ${ }^{11}$

En Chile se realiza vigilancia epidemiológica de las IIH desde 1982. El sistema de vigilancia epidemiológica de las IIH incluye desde 1996 la observación de las infecciones por tipos específicos de cirugía. El sistema tiene cobertura nacional y es obligatorio para todos los hospitales.

Se presentan en esta publicación los resultados de la vigilancia epidemiológica de infecciones en cirugía de implantes de prótesis de cadera notificadas por los hospitales chilenos entre 1996 y 1999.

\section{METODO}

La vigilancia de operaciones de prótesis de cadera es obligatoria para los hospitales que realizan más de 40 operaciones de este tipo al año, aún cuando los hospitales que realizan menos operaciones también pueden notificar sus IIH. La información sobre las infecciones se obtuvo con la revisión de las historias clínicas de pacientes con operaciones de colocación de implantes de prótesis de cadera parcial o total. La revisión es realizada durante la hospitalización o reingreso del paciente al hospital por las enfermeras de IIH, de acuerdo a los procedimientos habituales de la vigilancia epidemiológica. ${ }^{12}$ No se revisan rutinariamente las historias clínicas de los pacientes en control ambulatorio. La definición utilizada es "presencia de pus en el sitio de la incisión quirúrgica con o sin cultivos microbiológicos positivos" o "diagnóstico médico de infección, registrado en la historia clínica". La información acumulada se envía trimestralmente al Programa de IIH del Ministerio de Salud en formularios ad hoc. El sistema de vigilancia epidemiológica vigente no aporta la información necesaria para ajustar tasas por factores de riesgo de los pacientes.

\section{RESULTADOS}

Incidencia. Se recibió información de las operaciones realizadas entre 1996 y 1999 en 28 hospitales, 24 de ellos del sector público. Se notificaron los resultados de la vigilancia de 153 trimestres/hospital, con 134 infecciones en 
3.889 operaciones y una tasa acumulada de $3,5 \%$, (intervalo de confianza de $95 \%$ [IC95\%] entre 2,9 y $4,1 \%)$. Un establecimiento, el Instituto de Traumatología, aportó 30,3\% de las operaciones y $33,6 \%$ de las infecciones en este estudio. No hubo diferencias significativas entre las tasas crudas del Instituto Traumatológico, $3,8 \%$, y la de los hospitales generales con tasa de $3,3 \%$ (riesgo relativo [RR]: 1,15 con IC $95 \%$ entre 0,82 y $1,65, \mathrm{P}: 0,4)$ o del subgrupo de los hospitales generales con más de 100 operaciones cuya tasa fue de $2,7 \%$ (RR: 1,41 con IC $95 \%$ entre 0,95 y $2,04, \mathrm{P}: 0,08)$. No hay una tendencia de las tasas a aumentar o disminuir en el transcurso de los años analizados (Tabla 1). Las tasas fueron significativamente mayores en los hospitales con menos de 20 operaciones trimestrales promedio (tasa 5,0\%) comparadas con los hospitales con 20 o más operaciones trimestrales promedio (tasa 2,9\%) con un riesgo relativo de 1,72 (IC 95\% entre 1,26 y 2,47, P: 0,0009) (Tabla 2). Sólo se obtuvo información de las infecciones, de acuerdo al tipo de artroplastía, en el Instituto Traumatológico, institución en que no se observó diferencia de la tasa en artroplastías totales, $4,1 \%$, con respecto a $3,4 \%$ en artroplastías parciales (RR: 1,23 con IC95\% entre 0,68 y 2,24 y P: 0,5$)$.

Microbiología. La información microbiológica corresponde a los hospitales generales, en el período 1997 - 1999 en que la información es completa. Se identificó al menos un agente etiológico en $60,8 \%$ de las infecciones, con una gran variedad de agentes, predominando los bacilos Gram negativos no fermentadores (33,3\%): Acinetobacter baumannii y

Tabla 1. Tasa de infecciones quirúrgicas en operaciones de implantes de prótesis de cadera.

Chile 1996-1999

\begin{tabular}{lcccc}
\hline Año & Infecciones & Operaciones & Tasa (\%) & IC 95\% \\
\hline & & & & \\
1996 & 24 & 551 & 4,4 & 3,0 a 6,6 \\
1997 & 31 & 1.260 & 2,5 & 1,8 a 3,6 \\
1998 & 39 & 928 & 4,2 & 3,1 a 5,7 \\
$1999 *$ & 40 & 1.150 & 3,5 & 2,6 a 4,8 \\
Total & 134 & 3.889 & 3,5 & 2,9 a 4,1 \\
& & & & \\
\hline
\end{tabular}

Tabla 2. Tasa de infecciones quirúrgicas en implantes de prótesis de cadera, de acuerdo al número de intervenciones quirúrgicas. Chile 1996-1999

\begin{tabular}{|c|c|c|c|c|c|}
\hline Volumen quirúrgico & Hospitales & Infecciones & Operaciones & Tasa $(\%)$ & IC $95 \%$ \\
\hline $\begin{array}{l}\text { Bajo } \\
<20 \text { operaciones/trimestre }\end{array}$ & 22 & 54 & 1.079 & 5,0 & 3,8 a 6,5 \\
\hline $\begin{array}{l}\text { Alto } \\
\geq 20 \text { operaciones/trimestre }\end{array}$ & 6 & 80 & 2.810 & 2,9 & 2,3 a 3,5 \\
\hline Total & 28 & 134 & 3.889 & 3,5 & 2,9 a 4,1 \\
\hline
\end{tabular}


Tabla 3. Etiología de las infecciones de implantes de prótesis de cadera en hospitales generales. Chile 1997-1999*

\begin{tabular}{lcr}
\hline Total infecciones 1997-1999 & \multicolumn{2}{c}{79} \\
Con agente etiológico identificado & $48(60,8 \%)$ \\
\hline Microorganismo & $\mathbf{n}$ & $\mathbf{( \% )}$ \\
\hline Acinetobacter baumannii & 14 & 22,2 \\
Staphylococcus aureus & 11 & 17,5 \\
Pseudomonas spp & 7 & 11,1 \\
Escherichia coli & 5 & 7,9 \\
Klebsiella pneumoniae & 4 & 6,3 \\
Staphylococcus coagulasa negativa & 4 & 6,3 \\
Proteus spp & 3 & 4,8 \\
Otros (1 caso cada uno) & 15 & 23,9 \\
& & \\
Total & $63 * *$ & 100,0 \\
& \multicolumn{3}{c}{} \\
** :en algunas infecciones se identificó más de una especie. \\
\hline
\end{tabular}

Pseudomonas spp, mientras que Staphylococcus coagulasa negativa y $S$. aureus constituyeron, en conjunto, $23,8 \%$ de los agentes. (Tabla 3).

\section{DISCUSION}

Las infecciones de prótesis de cadera son un problema importante por causar morbilidad pues con frecuencia requieren su recambio o retiro que pueden resultar en invalidez permanente grave, en particular por tratarse en su mayoría de pacientes de edad avanzada. Además aumentan los costos por hospitalización prolongada, reintervenciones, uso de antibióticos, rehabilitación y licencias médicas, entre otros.

En el país es aún muy reciente la vigilancia epidemiológica de infecciones asociadas a cirugía de implantes de cadera. Sin embargo, las tasas observadas son elevadas comparadas con las comunicadas en la literatura norteamericana que, en general son de $2 \%$ o menos, considerando el tiempo total de uso de la prótesis desde su instalación hasta su retiro o la muerte del paciente. Es posible que los datos nacionales presentados tengan subnotificación de las infecciones, y por lo tanto la tasa real puede ser aún mayor, dada por la sensibilidad propia del sistema de vigilancia y porque se limitan principalmente a lo observado durante la hospitalización o el período post egreso más inmediato.

Los agentes etiológicos identificados difieren substancialmente a los citados en la literatura. En Chile, los agentes predominantes en esta vigilancia han sido bacilos Gram negativos no fermentadores (BNF) mientras que en las publicaciones extranjeras predominan las cocáceas Gram negativas, principalmente $S$. aureus y S. coagulasa negativa. En nuestros datos, S. aureus y S. coagulasa negativa representan sólo el $23,8 \%$ de los agentes identificados.

De igual forma, en infecciones de la herida operatoria de cirugías consideradas "limpias" operaciones que no involucran implante -predomina en nuestro medio $S$. aureus $(53,9 \%)$. En las infecciones de herida operatoria vigiladas desde 1996 (cesáreas, hernias inguinales sin colocación de mallas, colecistectomías laparoscópicas y por laparotomía), S. aureus y S. coagulasa negativa constituyen más del $40 \%$ de los agentes identificados y los BNF son menos del $4 \% .^{13}$

Es posible que el predominio de BNF en las operaciones de implantes de cadera, con alta frecuencia de identificación de A. baumannii, sea consecuencia de la colonización de la piel por una prolongada hospitalización pre operatoria.

Este panorama etiológico de las infecciones en implantes de prótesis de cadera debe ser considerado para establecer los esquemas de antibioprofilaxis más adecuados.

$\mathrm{Al}$ igual que en publicaciones previas, se observa una relación inversa de las tasas de IIH de acuerdo al número de intervenciones quirúrgicas: a mayor volumen quirúrgico del hospital, menores son las tasas de IIH. Este hecho puede deberse a distintos factores, tales como la experiencia de los cirujanos, calidad de la técnica quirúrgica en los centros traumatológicos con mayor número de intervenciones quirúrgicas y diferencias en los factores de riesgo de los pacientes, entre otros, los que deben ser estudiados.

Los hallazgos de la vigilancia epidemiológica pueden deberse a múltiples factores, entre otros a que el sistema de vigilancia en uso no distin- 
gue entre infecciones superficiales y profundas que involucran la prótesis ni entre infecciones precoces y tardías. Además las tasas reales pueden ser mayores a las observadas por una subnotificación y escaso seguimiento de los pacientes operados, con posterioridad al egreso. Estos hechos son fundamentales para evaluar el impacto en morbilidad de las elevadas tasas y las particularidades de su microbiología, pues es posible que las infecciones tardías tengan agentes etiológicos diferentes.

El impacto económico de las infecciones de prótesis de cadera es importante. Si el promedio de prolongación de hospitalización es mayor a 27 días en el período estudiado en los hospitales generales, estas infecciones han sido causa de un exceso de hospitalización de alrededor de 3.700 días. A esta información se debe agregar el costo en tratamiento, rehabilitación, reintervenciones quirúrgicas y de las prótesis que es necesario retirar.

La vigilancia epidemiológica tiene por objetivo identificar problemas que requieren intervenciones específicas o mayor investigación. En el caso de las infecciones en cirugía de implantes de prótesis de cadera permite reconocer un problema importante que debe ser estudiado con mayor detalle, dado que las tasas observadas son mayores que las publicadas en países de mayor desarrollo económico, un panorama etiológico muy diferente al publicado y al observado en el país en otros tipos de operaciones consideradas "limpias".

Se requiere mayor estudio sobre el tipo de infecciones y sus factores de riesgo en el país, que permitan orientar medidas preventivas. Es de interés definir los factores de riesgo para infecciones por BNF, que pudieran ser distintos a los asociados a infecciones causadas por otros agentes, en particular el tiempo de hospitalización preoperatorio, que podría favorecer la colonización por A. baumannii. También es necesario investigar sobre las infecciones tardías, información que no es conocida cabalmente en el país. Dado el bajo número de infecciones por hospital, con excepción del Instituto Traumatológico ningún centro tuvo más de 7 infecciones por año. Para contar con la información necesaria en un período de tiempo breve, los estudios se pueden realizar en forma restrospectiva o con carácter multicéntrico, especialmente si se planifican en forma prospectiva.

\section{RESUMEN}

La vigilancia epidemiológica de las infecciones en cirugía de prótesis se estableció en los hospitales chilenos a partir de 1996. Entre 1996 y 1999 se notificaron 134 infecciones en 3.889 operaciones - información proveniente de 28 hospitales- con una tasa acumulada de infecciones de 3,5\%. La vigilancia epidemiológica en Chile no diferencia infecciones de la herida operatoria de las de implantes propiamente tal y el período de observación se limita a la hospitalización y/o al período post hospitalización más inmediato. En esta casuística hubo predominio de bacilos Gram negativos no fermentadores $(33,3 \%)$ principalmente Acinetobacter baumannii. Las cocáceas Gram positivas representaron el 23,8\% de los agentes etiológicos. Se observó que a mayor volumen de operaciones menor es la tasa de infecciones $(5,0 \%$ en hospitales con bajo número de intervenciones quirúrgicas y $2,9 \%$ en hospitales de alto volumen quirúrgico). Los hallazgos observados sugieren factores de riesgo que deben ser estudiados, como el tiempo de hospitalización preoperatorio y el grado de experiencia de los centros quirúrgicos.

\section{BIBLIOGRAFIA}

1.- LEW DP, PITTET D, WALDVOGEL F A. Infections that complicate the insertion of prosthetic devices. En Hospital Epidemiology and Infection Control. Mayhall G. editor. Williams \& Wilkins 1996 pp: 731-48.

2.- CULVER D H, HORAN T C, GAYNES R P et al. Surgical wound infection rates by wound class, operative procedure, and patient risk index. Am J Med 1991; 91 (Suppl 3B): 1525-75.

3.- FERNANDEZ-ARJONA M, GOMEZ-SANCHA F, PEINADO-IBARRA F, HERRUZO-CABRERA R. Risk infection factors in the total hip replacement. Review of 873 patients. Eur J Epidemiol 1997; 13 (4): 443-6.

4.- BECK-SAGUE C M, CHONG W H, ROY C, ANDERSON R, JARVIS W. Outbreak of surgical wound infections associated with total hip arthroplasty. Infect Control Hosp Epidemiol 1992; 
13: 526-34.

5.- LIENDO C, PAULOS J, MENDOZA S, CAMACHO

M. Infecciones en artroplastía de cadera. Rev Chil Ortopedia Traumatol 1997; 3-4: 98-106.

6.- POSS R, THOMHILL T S, EWALD F C, THOMAS W H, BATTE N J, SLEDGE C B. Factors influencing the incidence and outcome of infection following total joint arthroplasty. Clin Orthop 1984; 182: 11726.

7.- POSS R, MALONEY J P, EWALD F C et al. Six to eleven years result of total joint arthroplasty in rheumatoid arthritis. Clin Orthop 1974; 182: 10916.

8.- STECKELBERG J M, OSMON D R. Infections associated with indwelling medical devices. Washington American Society of Microbiology Press, 1994: 259-90.

9.- SHEPPEARD H, CLEAK D K, EARD D J,
O'CONNOR B T. A review of early mortality in elderly patients following Charnley total hip replacement. Orthop Trauma Surg 1980; 97: 243-8.

10.- HAVELIN L I, ESPEHAUG B, VOLLSET S E, ENGESAETER L B. Early failures among 14.009 cemental and 1.326 uncemented protheses in primary cos-arthrosis. Acta Orthop Scand 1994; 65: 1-6.

11.- KREDER H J, DEVO R A, KOEPSELL T, SWIONTKOWSKI M F, KREUTER W. Relationship between the volume of total hip replacements performed by providers and the rates of postoperative complications in the state of Washington. J Bone Surg Am 1997; 79: 485-94.

12.- OTAIZA F, BRENNER P. Sistema de vigilancia de las infecciones intrahospitalarias, Ministerio de Salud, 1998.

13.- OTAIZA F, BRENNER P. Informes de la vigilancia epidemiológica de las infecciones intrahospitalarias en Chile 1994-1996, 1997, 1998. Ministerio de Salud de Chile.

Correspondencia a:

Fernando Otaíza O'R.

Unidad de Infecciones Intrahospitalarias

División de Salud de las Personas

Ministerio de Salud.

Av. Mac Iver 541, Santiago, Chile.

Fono: 56 (3) 6300476

E-mail: fotaiza@minsal.cl 